\title{
Collaborative Portal for Supporting Manufacturing Resources Selection in a Global Market
}

\author{
Vaibhav Shah ${ }^{1}$, Maria Leonilde Rocha Varela ${ }^{1,2}$, Goran D. Putnik ${ }^{1,2}$ \\ ${ }^{1}$ ALGORITMI Research Centre, University of Minho, Guimarães, Portugal \\ vaibhav.shahedps.uminho.pt \\ ${ }^{2}$ Department of Production and Systems, University of Minho, Guimarães, Portugal \\ \{leonilde, putnikgd\} @dps. uminho.pt
}

\begin{abstract}
In the last decades the manufacturing industry has shifted from largely single location centralized manufacturing towards networked manufacturing spread over multiple sites. Companies have either established new production sites in multiple locations or established strategic partnerships to respond to to market changes more rapidly as well as for more efficient and effective sharing of resources, through the so-called integrated manufacturing systems environments. In this context, proper collaboration among different locations and/or partners becomes more critical. Thus, development of appropriate integrated tools, to enable clients or network partners to accurately select appropriate manufacturing resource from the widened global market context, becomes emergent. Therefore, this work is aimed at providing a contribution to maximize overall benefit of businesses, in the context of a networked scenario, and subject to a set of configurations and restrictions on part of the manufacturing resources providers and searchers. Thus, the authors propose a collaborative portal to enable to perform a better manufacturing resources selection, based on technologies integration for supporting accurate orders processing.
\end{abstract}

Keywords: Collaborative Portal, Ubiquitous Manufacturing System, Global Market, Resources Selection.

\section{Introduction}

Collaborative work environments have rapidly emerged recently, from various contributions to ensure more efficient decision-making in manufacturing systems management, to fulfil functional and other requirements of globally spread companies. The present work proposes a web-based Portal for supporting collaborative manufacturing resources selection within a global market scenario in the context of a Ubiquitous Manufacturing System (UMS). Hence, an interactive portal, with a peer-to-peer (P2P) network based architecture, using web services and other Internet based technology offering support for resources selection for orders processing is proposed. The collaboration functionality is supported by several tools provided for handling such condi- 
tions. The purpose of the presented work is to describe the proposed collaborative portal to enable full potential of the network-based manufacturing systems in order to facilitate information sharing as well as collaboration among globally distributed users [1].

The web portal underlying this work includes a set of functionalities throughout several peers in the network, which holds manufacturing resources for processing different kind of orders. The integrated tools enable to more easily identify manufacturing resources that are available for processing orders, to be delivered according to a set of specified requirements. These requirements include due dates satisfaction, along with other more specific ones, namely related to processing and quality functional requirements, as well as conditions imposed on logistics, costs and payments processing, while considering other restrictions imposed by producers, related to their manufacturing resources, namely regarding resources availability and corresponding time restrictions and consequent due dates negotiation.

The rest of this paper is organized as follows. In section 2 a brief contextualization about a ubiquitous manufacturing system is provided. Section 3 refers to collaborative manufacturing resources selection within the UMS context. In section 4, a portal for technologies integration is given, as well as the underlying data model for manufacturing resources selection and web-based communication data exchange. Moreover, the web-based interface for supporting the resources selection problem solving is also illustrated, through a use-case. Finally, in section 5, some more discussion and concluding remarks are given.

\section{Ubiquitous Manufacturing System}

Ubiquitous Manufacturing System (UMS), at a higher level, can be seen as a platform to connect people, knowledge, processes, decision-making and data (information). In this context, it is our conviction that through a UMS, innovation plays a fundamental role for Virtual Enterprise (VE) integration. The UMS environment's characteristic is that it is possible to enable a system of distributed, manufacturing sub-systems or cells, integrating the corresponding technologies for management support, which work in a collaborative interconnected way, under the supervision of a market-ofresources (MR) manager, who controls the entire UMS, which includes connections among clients, that may or may not belong to the Meta-Organization (MO) environment. Here, brokering services play an important role too, to accomplish an integrated real-time collaborative management, in the true sense, of the whole networked/distributed manufacturing environment [2]. Here a decision-supported manufacturing resources selection process is an important functionality.

Fig. 1 shows an outline of UMS network environment. Here, it's important to enable co-innovation rather than focusing on individual innovation. This kind of innovation can be enabled by technology integration, which can also be seen as a commodity. Hence giving a real shape to an abstract concept of UMS and with the presented work it is intended to contribute in this direction, by enabling tecnologies 
integration to support selection of resources for collaborative manufacturing in a global MR [2].

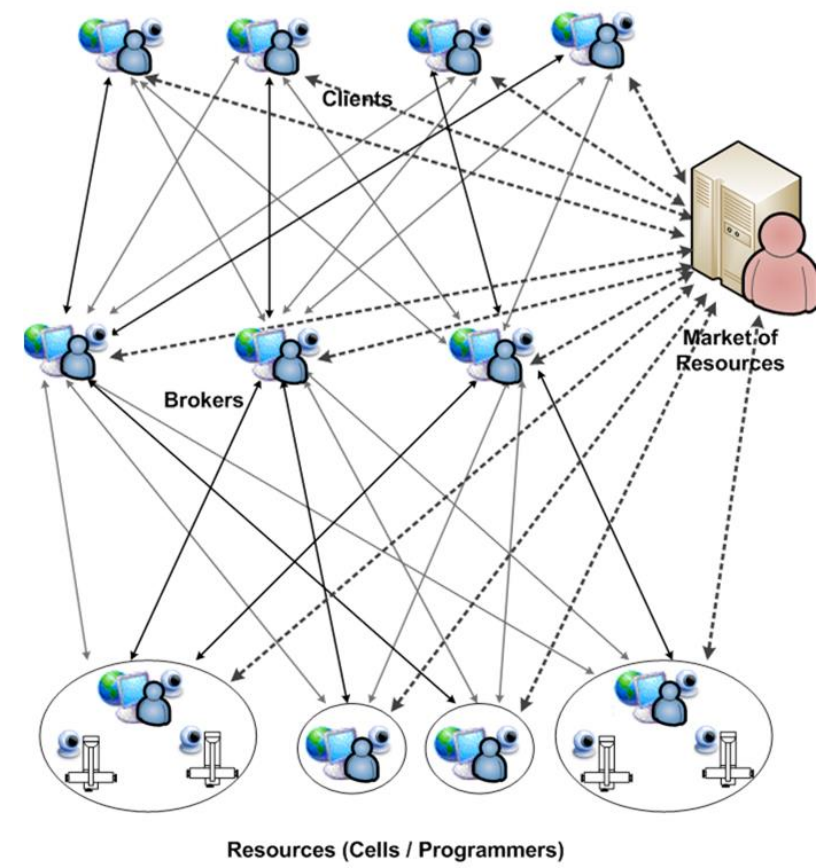

Fig. 1. Meta-organizational environment of a ubiquitous manufacturing system $[3,4]$.

\section{Collaborative Resources Selection in a UMS}

Manufacturing resources selection (MRS) issues are strategic in nowadays innovative enterprises that are expected to provide products under an unpredictably changing market. Considering a distributed manufacturing environment, enterprises have to form alliances with suppliers, other business partners \& customers, and then, one enterprise often takes care of only a part of the entire production process [5]. Because of this, problems related to MRS should be considered decentralized decision processes [2]. In other words, to support the decentralized MRS, an information infrastructure is necessary, namely for coordination, collaboration as well as communication among different partners or enterprises [6]. Manufacturing resources selection is a widely investigated topic along the past two-three decades and currently, in the context of VE and Global Markets it plays a critical role as it enables to fulfil the requirements of this type of network based manufacturing environments [7, 8].

Acritical success factor to consider while implementing Collaborative Manufacturing Resources Selection (CMRS) is the system's possibility to deal with different constraints, regarding each of the manufacturing processes, since different characteristics of a manufacturing system result through a set of imposed constraints, through- 
out the concerned manufacturing processes. Though it's not entirely possible to classify and consider all different constraints, some major categories can be defined and integrated through a rather simple schema, and some important ones of these are briefly described below (as given in [2]).

Precedence Constraint: These constraints are well-known in MRS and scheduling problems. In forward scheduling, a job can be started only after all of its predecessor jobs are completed. On the other hand, in backward scheduling, a job must be completed before all of its successor jobs are started. All jobs have events such as start \& completion. Additionally, there are other independent events such as products shipping, arrival of materials or parts, change or beginning of production shifts and so on. Any of these events can have a precedence constraint also. Moreover, precedence constraints have several variations according to manufacturing processes characteristics, e.g., within some scenarios a precedence constraint with min. or max. interval time may occur. Another case may be, for instance, that two jobs could be overlapped for a particular timeframe, due to continuous manufacturing processes. This can also be included in a certain category of types of precedence constraints.

Switching Constraint: If two jobs are executed over the same resource, then they have an exclusive relation, i.e. one job should be started after another is finished. Hence, the two cannot be executed at the same time. The proposed model includes this constraint - which may have many variations too. Generally, practical MRS and scheduling problems deal with switching constraints in a context, in which constraints are predefined according some engineering reasons. For instance, one job can be started after some time-period, after the end of another one, or a case where one job should be begun just after another is finished. Another practical case of use of this constraint happens due to changing interval setup-time, depending on the combination characteristics of two jobs.

Stock Constraint: Stock level of an item/component can change during the scheduling time period. Every type of items, such as finished products, work-in-progress inventory, components and raw materials may have some stock. Hence, stock constraints can be defined where the stock-level should be more than a min. level value and less than a max. level value. Normally, safety stock levels are defined just as max. stock levels, which may be related to space constraints of buffers and/or to the manufacturing politics used, such as just-in-time (JIT).

Loading Constraint: These constraints deal with the items' levels. These kinds of constraints concern about a usage level of charged resources, during the planning horizon. Thus, the level of jobs related resource usage allocated to that resource at that time should be within a predefined range between a min. and a max level. A maximum level is defined as availability of the resource performance in this type of constraint. The allowable range of the constraint may vary from 0 to 1 , if the target resource is occupied, and the level of usage may take either 0 or 1 . The maximum and the minimum levels of each loading constraint are static sometimes, but variable usually, during the planning period, for example, due to a changing number of workers on part-time regime.

State Constraint: They restrict the execution of jobs or events in a general form. This type of constraints is represented using pre-conditions \& post-conditions, for 
execution of particular events or jobs. Post-conditions perform as actions of events or jobs. These conditions are defined through primitives attributes and some mathematical expressions. These primitives attributes are altered during the planning timeline, by the execution of the events or jobs arising from allocation of resource and schedule plan.

Fig. 2 shows an illustrative interface for supporting businesses or clients to select appropriate resources for processing orders according to some imposed constraints, from a list of resources available through a P2P network of globally distributed resources.

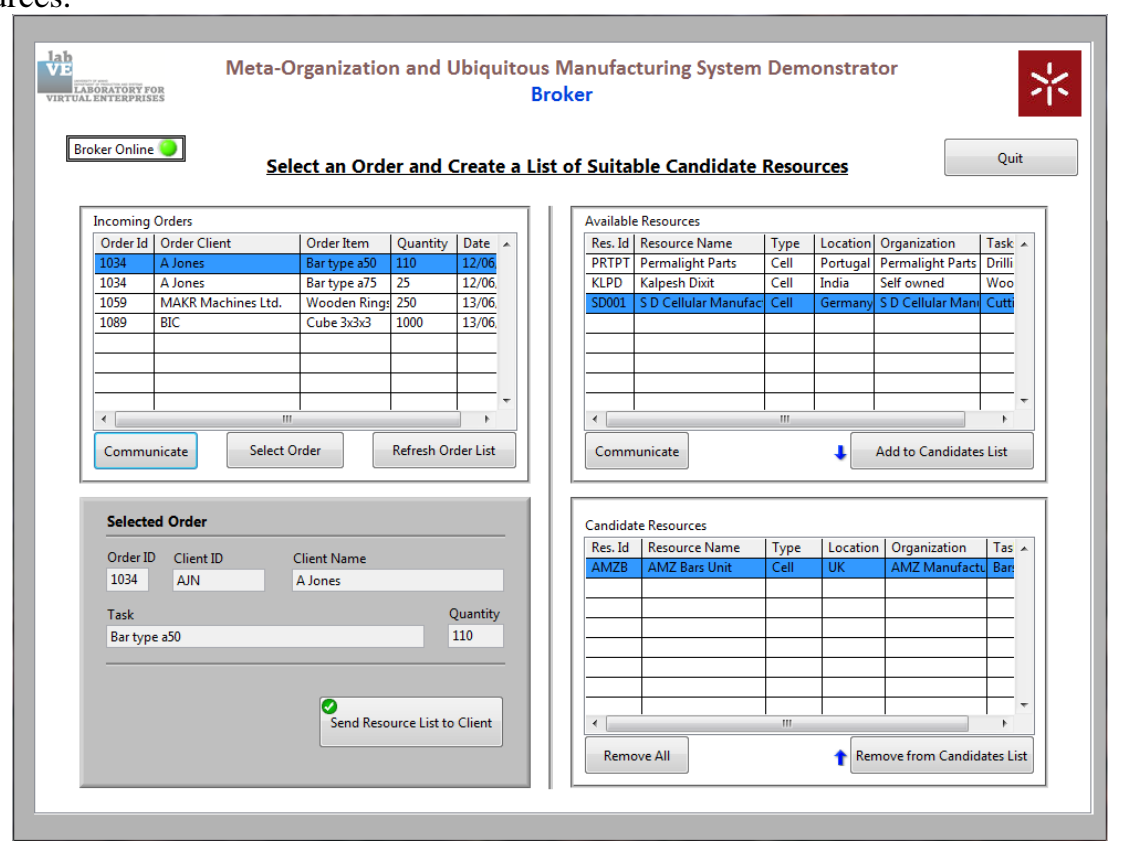

Fig. 2. Interface illustration for supporting resources selection.

The order related information, as illustrated in Fig. 2, has to be automatically generated, according to the information provided by each business, in real-time and with dynamically updated information regarding each production plan obtained through each business methods and approaches for Collaborative Manufacturing Scheduling Resources Selection and Scheduling (CMRSS). Therefore, the framework put forward in this paper, for supporting CMRSS problems resolution aims at providing an easy integration of legacy systems existing on each business, belonging to the $\mathrm{P} 2 \mathrm{P}$ network, offering the possibility of different technology and back-end systems integration, in order to facilitate the corresponding decision-making processes.

Moreover, the collaborative approach proposed includes a brokering service for enabling further refinement and adjustments to the ordering plans, based on human interactions, which are enhanced through diverse kinds of media-based communications put available through the underlying portal that is described next. 


\section{$4 \quad$ Portal for Technologies Integration}

Collaborative manufacturing resources selection and scheduling (CMRSS), in terms of optimal plans, means finding the best solution absolutely, for a problem. But practical CMRSS problems, as encountered in real world, normally do not reach such an optimal solution, since most of these deal with a lot of constraints, as described above, which only enables to arrive at some feasible solutions. Furthermore, usually it is hard to see an explicit relationship between a desired solution and its appropriate solving action. Hence, different optimization procedures can also be used, to try to find a better solution.

In the presented paper the authors propose an integrated decentralized CMRSS approach, through combining a different kind of technology, including back-office applications, for instance based on, Visual Basic programs and excel or simulationbased ones, which are usually locally used businesses.

Moreover, the underlying problem data representation schema permits users to add their own domain specific constraints. These constraints are later on used through dispatching rules, namely when a resource of a (manufacturing) system is ready to start, and that there are more than one jobs ready to begin on a given resource. The dispatching rules are part of the proposed CMRSS approach. They are used as a step on the global CMRSS approach. Therefore, there are two main approaches. One that is based on an automatically generated solution, usually performed as a first step of the whole CMRSS process, based on some kind of back-office application or some other approach, based on heuristics or meta-heuristics, such as genetic algorithms (GA), simulated annealing or "Tabu search" or even other simulation procedures based approaches. And another approach, usually at a second step of the CMRSS process is an approach, based on direct communication, through a brokering service. The latter doesn't directly focus on optimization, but it's very practical and important for real-world problems solving, e.g. for final adjustments of ordering plans in the context of the UMS. Therefore, the proposed system approach enables various types of procedures and technologies integration for supporting CMRSS.

\subsection{Interfaces to interact with businesses}

Fig. 3 shows an interface for supporting the communication-based step of the CMRSS approach, typically used for the final refining and adjustments of the ordering plans through communication based on a complete media support between businesses and some broker available on the P2P network of users. The visual image of plans and schedules, which are managed in various locations, is important especially in a decentralized manufacturing system. If each manufacturing site has its own schedulers developed by various IT vendors, their production plans cannot be visualized by a common viewer normally, without particular adapting programs. This actually creates a huge effort on implementation of systems, and both, the cost as well as the risk of the system's useful functionality, will be increased. 
Using interfaces developed in a general way, enables end-users to get personalized plan viewers. This provides visualizing flexibility, for instance, distributed schedules, anywhere over the Internet. Therefore, this work is based on a general model for ordering and production plans and underlying important data for supporting decisionmaking and corresponding data representation and processing. In order to present the model better, an illustration of the proposed portal is provided through Figs. 4 and 5 . In Fig. 4 it is possible to see an interface for supporting human interaction, between some client and a business provider, and both can share and visualize important information for enabling a better understanding about ordering data, like orders detailed scheduling and product prices and due dates establishment. In Fig. 5 it is possible to see an interface about the execution of the Hungarian algorithm, for doing the assignment of each of a set of parts to be processed on a set of machines and corresponding graphics including planned starting and finishing times of orders on the corresponding processing machines.

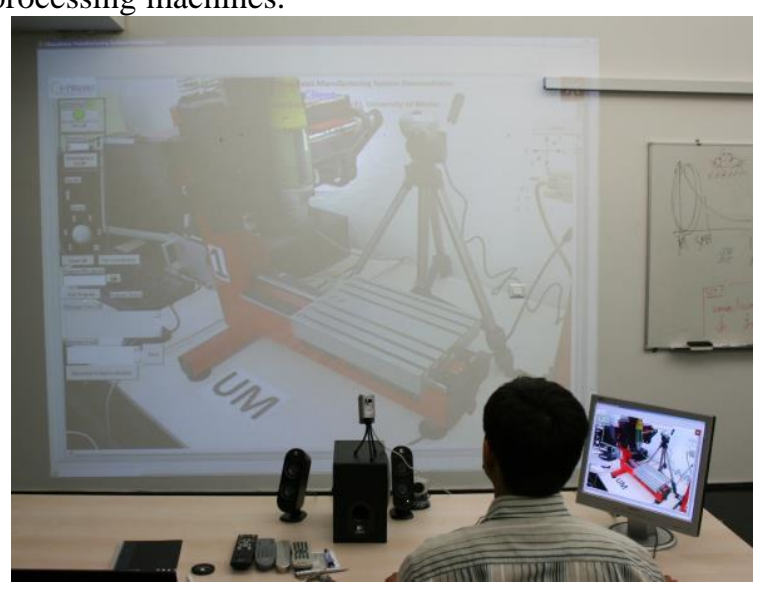

Fig. 3. Interactive portal.

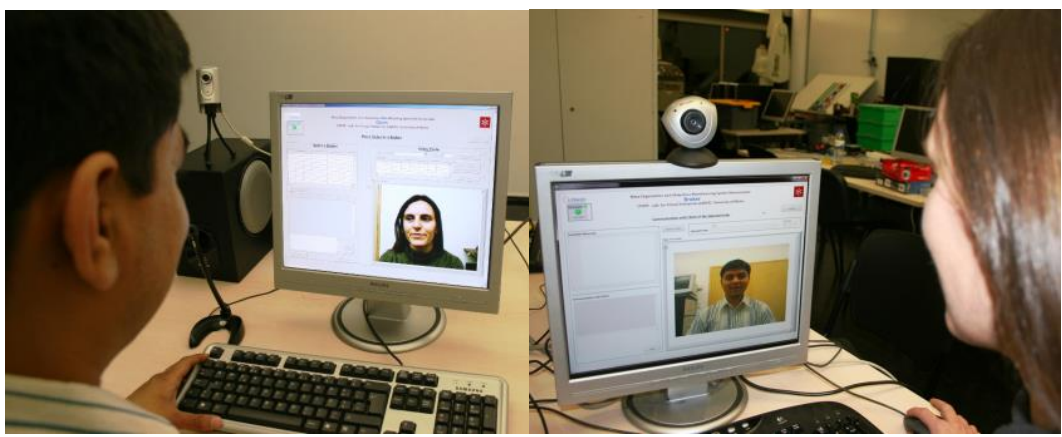

Fig. 4. Interface illustration - resources selection supported by a broker.

To contribute to a specification standard for CMRSS problems, the data is given through XML, based on a data structure which can be easily visualized through the Internet. In Fig. 5 a chart about a scheduling plan obtained is given. It gives an effec- 
tive visualization tool. Furthermore, by using XML based problem specifications, another type of charts, namely the Gantt charts, can easily be presented and visualized on the web through the proposed portal.

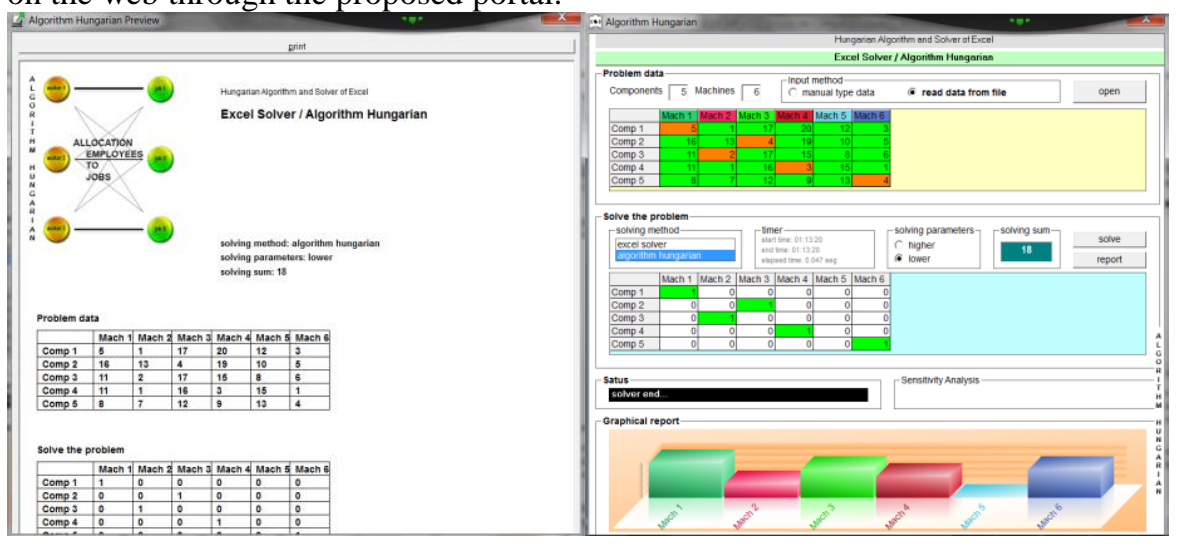

Fig. 5. Interface illustration - report with statistical data about orders processing.

\subsection{Distributed Knowledge Base and Control}

The proposed innovative approach includes a two step framework for fully enabling CMRSS. First, each business integrating a P2P network may use its own technology, as described above, to provide its ordering and production plans for being able to provide useful and accurate information, on a real-time basis, to other businesses on the network or to outside clients, based on its own legacy systems, which can be easily integrated through appropriate interfaces. Second, communication through a brokering service enables to establish final adjustments to ordering plans between businesses or between each business and outside clients. These brokering services are fully media-oriented and based on a distributed knowledge base for enabling to more easily share and dynamically update important information regarding CMRSS problems solving.

Moreover, the proposed approach and underlying portal also enables functional support for remotely controlling manufacturing devices, as illustrated through Fig.s 6 and 7.

The proposed portal is based on a standardized format to exchange data among different businesses. In manufacturing enterprises, there are various ICT applications including distinct planning \& scheduling software. In order to make a pliant communication between different applications, XML based data-modeling is used. A significant aspect of this approach is generation and visualisation of data by computers in proper and unique ways. Therefore, it's important to notice that the schema of data representation is general in order to fulfil the different kind of manufacturing enterprises requirements. 


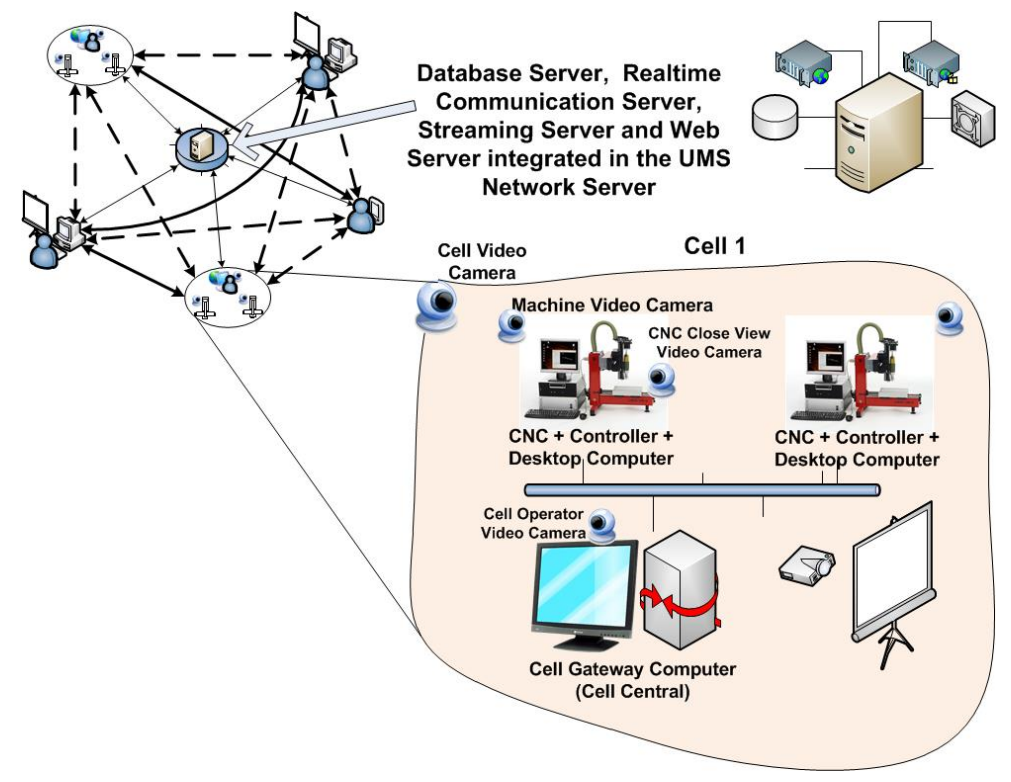

Fig. 6. Distributed knowledge base representation within the P2P network.

Regarding the management of networked manufacturing processes, CMRSS problems play a significant role. Hence, frameworks for supporting collaboration and communication between businesses enable to enrich business models, in the context of integrated businesses in the context of a UMS and global market.

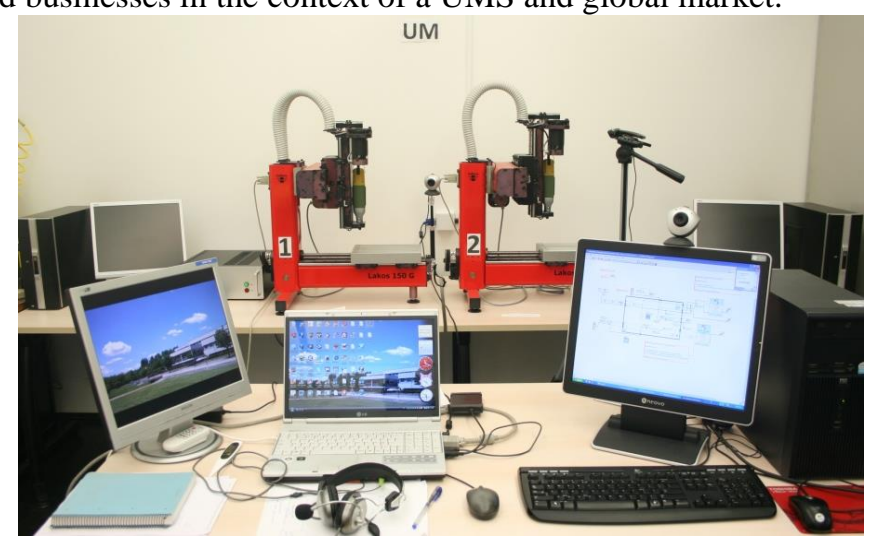

Fig. 7. Illustration of a remotely controlled machine cell.

\section{Conclusion}

This paper presented a portal for collaborative manufacturing resources selection and scheduling (CMRSS), which enables to easily and interactively communicate and 
establish orders among businesses and clients, based on an innovative approach and media-based communication. The innovative approach includes a two step framework for fully enabling CMRSS. Moreover, the proposed portal also enables functional support for remotely controlling manufacturing devices.

The effectiveness of the proposed integrated approaches was briefly described through some illustrations. A significant aspect of this approach is generation and visualisation of data by computers in proper and unique ways. It is necessary that the schema for data representation is general for different kinds of manufacturing enterprises requirements. Furthermore, collaboration also provides infrastructure for webbased application integration for all manufacturing enterprises, inside a Virtual Enterprise and Global Markets in a UMS environment.

\section{Acknowledgements}

The authors wish to acknowledge the support of the Foundation for Science and Technology - FCT, under the scope of the financed Project with reference: PEst20152020, as well as the FCT Doctoral grant referenced SFRH/BD/62313/2009.

\section{$7 \quad$ References}

1. Varela, L.R., Putnik, G.D. Ribeiro, R.A.: A web-based platform for collaborative manufacturing scheduling in a virtual enterprise, in Information and Communication Technologies for the Advance Enterprise, Vol. 2, pp. 87-108, Portugal (2012)

2. Vieira, G., Varela, M.L., Putnik, G.D.: Technologies Integration for Distributed Manufacturing Scheduling in a Virtual Enterprise, in Communications in Computer and Information Science: Virtual and Networked Organizations, Emergent Technologies and Tools, ViNOrg11, Portugal, Revised Selected Papers, Vol. 248, pp. 337-347, Springer Berlin Heidelberg (2012)

3. Putnik, G.D.: BM_Virtual Enterprise Architecture Reference Model. Technical Report RTCESP-GIS-2000-<GP-01>. Universidade do Minho, Portugal (2000)

4. Putnik, G.D.: BM_Virtual Enterprise Architecture Reference Model, in A. Gunasekaran (Ed.), Agile Manufacturing: 21st Century Manufacturing Strategy, pp. 73-93, Elsevier Science Publ., UK (2001)

5. Weiming, S., Lihui, W., Qi, H.: "Agent-Based Distributed Manufacturing Process Planning and Scheduling: A State-of-the-Art Survey", IEEE Transactions on Systems, Man and Cybernetics - Part C: Applications and Reviews, Vol. 36, No. 4, July (2006)

6. Herbsleb, J.D., Grinter, R.E.: "Splitting the organization and integrating the code: Conway's law revisited," icse, pp.85, 21st International Conference on Software Engineering (ICSE'99) (1999)

7. Wu, S.H., Fuh, J.Y.H., Nee, A.Y.C.: "Concurrent process planning and scheduling in distributed virtual manufacturing", IIE Transactions, Vol. 34, Issue 1, pp. $77-89$ (2002)

8. Hong, D., Li, C., Chentao, W., Qianni, D.: "A grid-based scheduling system of manufacturing resources for a virtual enterprise", The International Journal of Advanced Manufacturing Technology, Vol. 28, Ns. 1-2, pp. 137-141 (2006) 